\title{
Vehicle Routing Problem with Transshipment: Mathematical Model and Algorithm
}

\author{
Thanapat Leelertkij, ${ }^{1}$ Parthana Parthanadee $\mathbb{D D}^{2}$ and Jirachai Buddhakulsomsiri $\mathbb{D}^{1}$ \\ ${ }^{1}$ School of Manufacturing Systems and Mechanical Engineering, Sirindhorn International Institute of Technology, \\ Thammasat University, Khlong Luang, Pathum Thani, Thailand \\ ${ }^{2}$ Department of Agro-Industrial Technology, Faculty of Agro-Industry, Kasetsart University, Bangkok, Thailand \\ Correspondence should be addressed to Jirachai Buddhakulsomsiri; jirachai@siit.tu.ac.th
}

Received 1 June 2020; Revised 15 January 2021; Accepted 20 January 2021; Published 2 February 2021

Academic Editor: Alain Lambert

Copyright (C) 2021 Thanapat Leelertkij et al. This is an open access article distributed under the Creative Commons Attribution License, which permits unrestricted use, distribution, and reproduction in any medium, provided the original work is properly cited.

\begin{abstract}
This paper presents a new variant of vehicle routing problem with paired transshipment demands (VRPT) between retail stores (customers) in addition to the regular demand from depot to retail stores. The problem originates in a real distribution network of high-end retail department stores in Thailand. Transshipment demands arise for one-order-per-season expensive items, whose inventories at the depot may become shortage after the middle of a season, while they remain available at some retail stores. A transshipment demand is a request for items that need to be picked up from a specific store that has the items and delivered to the store that requests the items. The objective of solving the VRPT is to find delivery routes that can satisfy both regular demands and transshipment demands in the same routes without incurring too much additional transportation distance. A mixed integer linear programming model is formulated to represent the VRPT. Six small problem instances are used to test the model. A hybrid threshold accepting and neighborhood search heuristic is also developed to solve large problem instances of VRPT. The heuristic is further extended to include a forbidden list of transshipment demands that should not be included in the same routes. The purpose is to prevent incurring too much additional distance from satisfying transshipment demands. With the forbidden list, the problem becomes vehicle routing problem with optional transshipment demands (VRPOT). Computational testing shows promising results that indicate effectiveness of the proposed hybrid heuristics as well as the forbidden list.
\end{abstract}

\section{Introduction}

The vehicle routing problem (VRP) involves finding optimal routes for a fleet of delivery trucks, each with limited capacity, so as to minimize the total transportation distance. A vehicle routing network consists of one or more depots and customer nodes. Each customer has a request of items that can be satisfied by inventories at the depot. This type of request is called regular demand in this paper. A truck is then assigned to deliver the items to the customers within the load capacity limit of the truck, according to a delivery route. This paper extends the classical VRP to include transshipment demands. A transshipment demand is defined in this paper as the demand for items that the depot cannot directly satisfy. Instead, the demand can be satisfied by items at another node, i.e., a retail store. Incorporating transshipment demands, which requires picking up some items from one specific retail store and delivering them to another specific retail store, makes the problem become a VRP with transshipment (VRPT).

The VRPT is motivated by a real problem faced by one of the largest chains of retail department stores in Thailand. The retail chain has many store locations scattered around the capital city, Bangkok, and vicinity areas. These stores are served by a central warehouse (depot). At the stores, some merchandise are expensive, one-order-per-season items with a product life cycle of one year or less. Examples are brand name or designer products, fashionable clothes, and swimsuits. For these types of items, the depot places a large order from overseas before the selling season to serve the demand of all stores for the whole season. At the middle (and towards the end) of the season, inventories of these items would have already been distributed to all the retail stores, i.e., the depot no longer has the items. After that, transshipment demand 
arises when these items are sold out and become shortage at a store, but are still available at another store.

To better satisfy end customer demands, a sold-out store requests a transshipment of items from another store that has the items remaining in stock. This can satisfy the demand of the end customers that are willing to wait and satisfy future demand that may arise by balancing the inventory among different stores using transshipment. A transshipment requires two operations, picking up the item from a particular store and delivering the item to the store that requests the transshipment. After the transshipment demand is placed by the requesting store, the depot proceeds to identify a pickup node, i.e., a store that has the item. Thus, in the VRPT, the demand that each retail store places to the depot can be classified as (1) regular demand placed on a periodic basis that can be satisfied with items at the depot and (2) transshipment demand that must be satisfied with items at another store. Currently, transshipment items are relocated from the store that has the items (i.e., pickup node) to the store that requests the items (i.e., delivery node) through the depot. In other words, there are two operations when a truck visits the pickup node: (1) to deliver its regular demand and (2) to pickup the transshipment items. Then, the truck would bring the transshipment items back to the depot and deliver the items to the delivery node as part of the regular demand shipment in the next trip. This results in a delay of delivering the transshipment items to the requesting store and subsequently to the end customers, which may result in lost sales. It may also incur additional costs of handling and delivery to the end customers or a delayed pickup by the end customers at the store.

The depot has sought to improve this practice by designing delivery routes that can satisfy all or partial transshipment demands on the same delivery routes. The objective is to reduce the time to deliver the transshipment demand without incurring too much additional transportation distance. In other words, the depot attempts to make the item available faster at the requesting store and improve its customer services. Note that the merchandise is mostly expensive items for high-end customers. For such items, responsiveness is relatively more important than efficiency. An example of a network (Figure 1) demonstrates the delivery routes, where vehicles handle regular demand and a transshipment demand on the same trip.

From Figure 1, each customer node is set to be visited once by a vehicle. To satisfy the transshipment demand, the pickup node (node 6) and delivery node (node 7) are on the same trip, with the pickup node being visited before the delivery node. Every route starts and ends at the depot. The objective of solving the VRPT is to generate good delivery routes that can satisfy both regular and transshipment demands in the same delivery routes. In addition, a special case of VRPT, where the transshipment demand is optional, denoted as VRPOT, is also considered in this paper. Adding transshipment demand to the VRP will incur additional travel distance due to more restrictions on the delivery routes, i.e., pickup and delivery nodes must be visited in the same routes with an order of precedence. Making transshipment demands optional would allow the depot to decide which transshipment

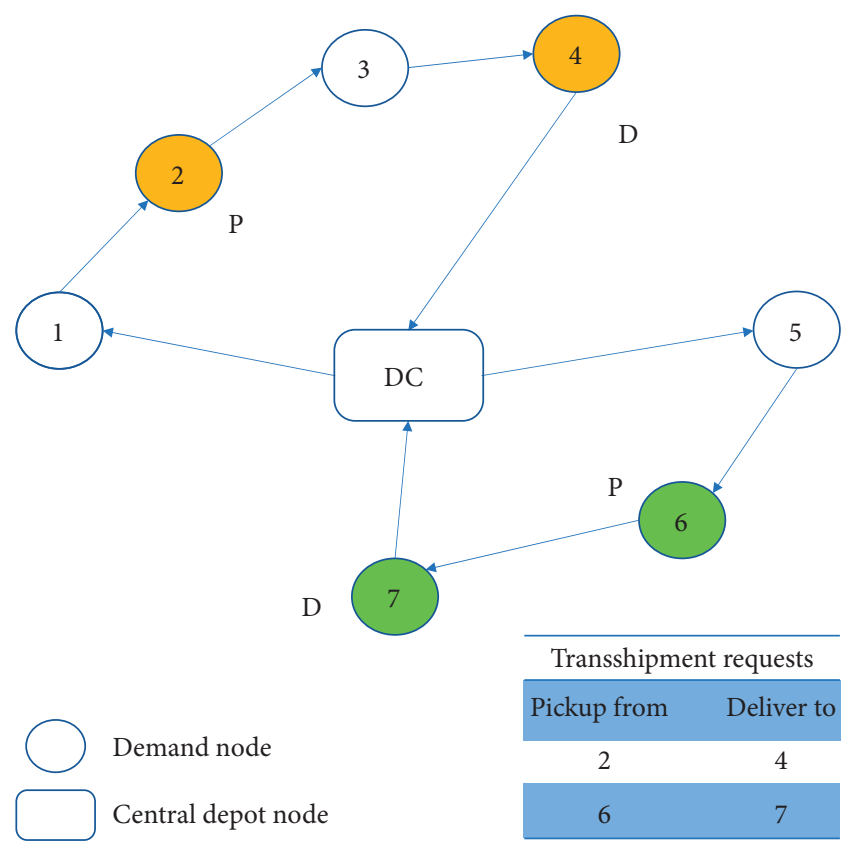

FIgURE 1: Vehicle routing problem with transshipment demand.

demand(s) should be satisfied without incurring too much additional distance. In a VRPOT, the unsatisfied transshipment demands would be picked up and brought back to the depot for the next delivery trip.

Significance of the VRPT is that the problem is originated in a real distribution network of depot to high-end retail stores in Thailand. To the best of our knowledge, previously studied VRP and its variants in the literature do not exactly match the problem imposed by the industrial user (depot) in this paper. The outcomes of this study not only fill a gap in the VRP literature (see next section) but also address the real-world problem to improve the current practice of an industrial user. In addition, impact of considering transshipment demand in a VRP is on reduction of delivery time as transshipped items are delivered on the same day as the regular demand. This is clearly beneficial to the retail store that requests the items because the items become available faster, which improves service to the end customers. It should be emphasized that in VRPT, the types of items that are requested for transshipment are expensive items. Most end customers prefer to purchase these item at the store, rather than purchasing them online.

The main contributions of this paper are threefold. First, we define a new VRPT that reflects a real problem found in the retail industry in Thailand. A mixed integer linear programming (MILP) model that represents the VRPT is formulated and demonstrated by solving small problem instances to optimality. The proposed model can be considered an improvement from a mixed integer model, which contains a nonlinear constraint, of a relatively similar problem, the one-to-one pickup-and-delivery problem with time windows in [1]. Second, a hybrid metaheuristic algorithm of threshold accepting (TA) and neighborhood search (NS), a so-called TA-NS heuristic in this paper, is developed to solve a medium to large-scale VRPT. Third, a component, 
called a forbidden list of transshipment pairs, is added to the TA-NS hybrid method to solve the VRPOT. Its purpose is to identify pairs of transshipment demands that should not be included on the same routes, to prevent incurring too much additional distance for satisfying the conflicting transshipment demands. The proposed TA-NS heuristics, without the forbidden list for VRPT and with the forbidden list for VRPOT, are extensively tested to compare their effectiveness. To summarize, the novelty of our research includes the newly defined VRPT and its MILP formulation, the developed TA-NS hybrid for solving large-scale problem instances, and the forbidden list that effectively balances between satisfying transshipment demands and transportation distance.

\section{Literature Review}

There is a vast literature on the VRP. Recent comprehensive reviews of the VRP and its variants can be found in [2]. The scope of the literature review given in this section focuses primarily on (1) single-depot VRP that features pickup and delivery or transshipment because overview of these problems can indicate the research gap that the VRPT under study aims to fill, (2) multiechelon, multicenter VRPs that consider transshipments, and (3) heuristic methods to solve these problems.

2.1. PDP, VRP with Pickup and Delivery, and VRP with Transshipment. An important relevant class of the VRP is the pickup and delivery problem (PDP). The PDP is a routing problem with different pickup locations and delivery locations for transporting goods (or people). For comprehensive reviews of the PDP, see [1]. The PDP can be classified into three types: many-to-many, one-tomany-to-one, and one-to-one, based on the pickup and delivery relations.

In a many-to-many PDP, each item may have multiple pickup nodes and delivery nodes, while any node can request pickup and delivery of the items. Ting et al. [3] introduced the MVSPDP, where some selected pickup nodes are chosen to supply all delivery nodes. The objective is to find minimal cost delivery routes for multiple vehicles with capacity and distance constraints. Xu et al. [4] considered a multiproduct, unpaired PDP that allows multiple visits to each customer, where more than one product can be delivered in a visit, but each product demand delivery cannot be split.

A one-to-many-to-one PDP is where the delivery operation is carried out from one depot to many customers, and the pickup operation is performed to bring items from many customers back to the depot [5-10]. The one-to-manyto-one PDP is also known as the VRPPD and its variants.

The VRPPD is an important extension of the VRP. In the VRPPD, there are two groups of customers: linehaul and backhaul. Linehaul customers request items to be delivered from the depot, while backhaul customers request items to be picked up and returned to the depot. The VRPPD has many variants classified by using the transportation behavior in response to the linehaul and backhaul customers. (i) VRPB (VRP with backhauls) specifies that vehicles must serve all linehaul customers before serving the backhaul customers $[8,10,11]$.

(ii) VRPMPD (VRP with mixed pickups and deliveries) allows vehicles to serve both linehaul and backhaul in any order $[7,12]$.

(iii) VRPSPD (VRP with simultaneous pickups and deliveries) is the problem where customers require pickup and delivery at the same time. Each customer is allowed to be served by only one vehicle that performs pickup and delivery, simultaneously $[5-7,9,13]$.

(iv) SVRPDSP (single vehicle routing problem with deliveries and selective pickups) by [14] is another variant of the VRPSPD. The difference is that the pickup operations can be omitted if they are not profitable.

(v) VRPDDP (VRP with divisible deliveries and pickups) may divide the delivery and pickup at a customer into two operations such that the customer may be visited twice either by the same vehicle or by two vehicles in different routes [15]. This relaxation makes it possible to create feasible routes, which would be infeasible in the VRPSPD, VRPB, and VRPMPD due to the fluctuation of loads. Note that "divisible" refers to separating delivery and pickup operations at one customer, which is different from partial delivery or pickup in split delivery VRP (see [16]).

(vi) Recently, Wassan and Nagy [17] further extended the VRPMPD and VRPDDP to include a restriction that deliveries and pickups can be mixed only if a certain amount of free space is available on the vehicle.

Finally, a one-to-one PDP consists of pickup and delivery requests in which each pickup location is specifically tied to one delivery location [18]. This type of problem can also be referred to as the dial-a-ride problem (DARP) for transporting people [19-22]. The transshipment demand, defined in this study, can be viewed as a one-to-one pickup and delivery request.

Recent studies on the one-to-one PDP that considered green aspects and other restrictions are [23, 24]. Madankumar and Rajendran [23] formulated two mathematical models for green VRPPD for a semiconductor supply chain. The first model focused on alternative fuel vehicles for the basic green semiconductor supply chain that has restrictions on product-vehicle compatibility and start and completion times and different request priorities for pickup and delivery. The second model was extended to handle having different fuel prices at different refueling stations so as to minimize the total cost of refueling and routing. Soysal et al. [24] proposed a mathematical model for a one-to-one pickup and delivery system with road segments. The proposed model considered the fuel consumption that is related to emissions, variable vehicle speed, and road categories (i.e., urban and nonurban) with different traffic regulations. The model was 
solved using a case study from the Netherlands. The results showed significant savings from adding these new factors.

Other relevant research studies to transshipment are [25-31]. Mues and Pickl [25] introduced the PDP with transshipment and time windows (PDPTTW) restriction, which is a one-to-one PDP that is extended to include transshipment points, where loads can be transshipped between transportation means that have different routes. Mathematical models for one transshipment location and arbitrary transshipment locations have been developed. Yang and Xiao [26] studied VRP with transshipment centers that serve as transfer points for arranging freight and storage from suppliers to customers. The decision for each vehicle is to choose between a route that travels directly to customer and a route that goes through transshipment centers. This concept of transshipment centers is not related to pickup and delivery of load.

Another relevant class of VRP is the inventory routing problem (IRP). In IRP, VRP is combined with inventory management problem such that pickups and delivery quantities from depot to customers are decided based on an inventory policy, which take into account the inventory holding cost. Coelho et al. [27] introduced the transshipment concept to IRP (the problem becomes IRPT), where items can be shipped to customers from the supplier or from another customer. Also, transshipments are performed by a subcontract carrier, which means that transshipment demands are not considered in the vehicle route from the depot. Azadeh et al. [28] later extended the IRPT to consider a single perishable product. With limitation of product shelf life, the complexity of the inventory management part is more complex.

Drexl [29] introduced the vehicle routing problem with trailers and transshipments (VRPTT). The problem originates in raw milk collection at farmyards, i.e., customers with given supply, where supplies are picked up by a set of heterogeneous vehicles stationed at one or several depots. Heterogeneous vehicles include autonomous vehicles, called lorries, that can move on their own, and nonautonomous vehicles, or trailers, that need to be attached to autonomous vehicle in order to move. The problem includes physical constraints that separate customers into a group accessible with lorry only and the other accessible with lorry and a trailer. In this problem context, transshipments only occur at transshipment locations on the routes to transfer load and couple and decouple trailers. Mathematical models of the problem were formulated and solved using branch-and-cut algorithms in [30].

Ahmad et al. [31] introduced a location routing inventory problem with transshipment (LRIPT), which integrates three logistics problems: location allocation, inventory management, and VRP, with additional decision of choosing a customer as a transshipment point. The transshipment point simply acts as a location where the supplier sends inventory to be distribute to other customers. A mathematical model of the problem and numerical examples to demonstrate the model are given.

Based on the above reviews, the research gap that the VRPT in this paper aims to fill can be seen in Table 1. From the table, it is clear that variants of VRP that consider transshipment demand with paired pickup and delivery are DARP and PDPTTW, but the two problems do not include the regular demand from the depot. While IRPT and LRIPT involve transshipment, the context is for selecting transshipment location, rather than transshipment demand of paired pickup and delivery. To the best of our knowledge, the definition of VRPT in our paper is the first that combines VRP (i.e., one-to-many-to-one PDP) for delivering items from depot to the customers and transshipment demands of paired pickup and delivery nodes of customers (i.e., 1-to-1 PDP) on the same route. In addition, our paper includes a variant of the problem, called VRPOT, where transshipment demand is optional and the unsatisfied transshipment demand is brought back to the depot (i.e., backhaul) to be dispatched to the delivery node on the next delivery route.

2.2. Multiechelon, Multicenter VRP and Extensions. Another class of VRP that is more complex is the twoechelon, multicenter VRP and its extensions. Some examples of recent works on MCVRP are [32-35]. The collaborative MCVRP was studied in [32] where collaboration is made among centers by reassignment of customers to centers (through clustering) as well as transshipments among centers. Wang et al. [34] then extended the problem so that collaboration can be made between multiple periods. A twoechelon location routing problem with simultaneous pickup and delivery was investigated in [33]. In this problem, delivery and pickup demands are unpaired (i.e., not tied to the same parcel), which is different from our problem. In addition, transshipments in the context of MCVRP occur among centers (i.e., distribution centers and logistics centers) in the first echelon, rather than among customers in the second echelon, which makes the problem settings different and not directly relevant to this paper.

An extension of MCVRP involves the delivery and pickup problem, which considers linehaul delivery and backhaul pickup of eco-package back to the recycling center [35]. In this problem, delivery requests are satisfied from a delivery center before pickup requests are satisfied to bring eco-package back to the pickup center, which makes the delivery and pickup request unpaired. Gansterer et al. [36] considered a multicenter pickup and delivery problem, where pickup and delivery requests are paired. The focus is on collaboration by redistributing the pickup and delivery request among centers (or carriers). Major differences from our paper are as follows: (1) capacity of the vehicles are not considered, which makes the problem become multicenter traveling salesman, and (2) there are no regular demands that request items from the depot in the problem.

2.3. Methods to Solve VRP and Extensions. Methods for solving the VRP can be categorized into exact algorithms and heuristic algorithms. The VRP is an NP-hard problem, which means that exact methods are not effective in solving medium to large problems. For such problem sizes, heuristics methods, which require much less computational time, but do not guarantee optimality, are good alternatives. 
TABLE 1: Research gap.

\begin{tabular}{|c|c|c|c|c|c|}
\hline Problem & Variant & Problem specific & $\begin{array}{l}\text { Linehaul } \\
\text { depot to D }\end{array}$ & $\begin{array}{l}\text { Backhaul P to } \\
\text { depot }\end{array}$ & $\begin{array}{c}\text { Transshipment } \mathrm{P} \\
\text { to } \mathrm{D}\end{array}$ \\
\hline Many-to-many PDP & MVSPDP & Each item is not specific to $\mathrm{P}$ and $\mathrm{D}$ nodes & & & Unpaired \\
\hline \multirow{6}{*}{$\begin{array}{l}\text { 1-to-many-to-1 PDP } \\
\text { (aka. VRPPD) }\end{array}$} & VRPB & Visit D nodes before $\mathrm{P}$ nodes & $\checkmark$ & $\checkmark$ & \\
\hline & VRPMPD & Any orders of $\mathrm{P}$ and $\mathrm{D}$ & $\checkmark$ & $\checkmark$ & \\
\hline & VRPSPD & Simultaneous $\mathrm{P}$ and $\mathrm{D}$ at each node & $\checkmark$ & $\checkmark$ & \\
\hline & SVRPDSP & $\mathrm{P}$ is selected only when profitable & $\checkmark$ & Selective & \\
\hline & VRPDDP & Customer node may be visited twice & $\checkmark$ & $\checkmark$ & \\
\hline & VRPTT & $\begin{array}{l}\text { Transshipments to transfer loads } \mathrm{b} / \mathrm{w} \text { vehicles at } \\
\text { transship points }\end{array}$ & & $\checkmark$ & \\
\hline \multirow[b]{2}{*}{ 1-to-1 PDP } & DARP & Each item is specific to a pair of $\mathrm{P}$ and $\mathrm{D}$ node & & & Paired \\
\hline & PDPTTW & $\begin{array}{c}\text { Transshipments between vehicles allowed at } \\
\text { transshipment points }\end{array}$ & & & Paired \\
\hline$I R P T$ & & $\begin{array}{l}\text { A mix of inv. management and VRP with } \\
\text { transshipment location }\end{array}$ & $\checkmark$ & & Not in VRP \\
\hline LRIPT & & $\begin{array}{l}\text { A mix of location, inventory, and VRP with } \\
\text { transshipment location }\end{array}$ & & & \\
\hline \multirow{2}{*}{ This paper } & VRPT & A mix of 1-to-many-to-1 PDP (regular demand) & $\checkmark$ & & Paired \\
\hline & VRPOT & and 1-to-1 PDP (transshipment demand) & $\checkmark$ & $\checkmark$ & Optional \\
\hline
\end{tabular}

Note. $\mathrm{D}$ denotes delivery node and $\mathrm{P}$ denotes pickup node.

A comprehensive review of the VRP and methodology can be found in $[2,37]$. The review given here focuses on recent heuristic algorithms for the PDP.

Two recent studies that involved variants of the manyto-many PDP are [3, 4]. Ting et al. [3] proposed three metaheuristics: genetic algorithm (GA), tabu search (TS), and scattered search (SS), to solve the so-called multivehicle selective PDP. The purpose of solving the MVSPDP is to find the shortest routes for vehicles that transport goods from some pickup nodes to all delivery nodes. Experimental results showed that TS outperformed GA and SS in terms of solution quality and computational time. $\mathrm{Xu}$ et al. [4] proposed an effective tabu search algorithm to solve a multiproduct, unpaired pickup and delivery vehicle routing problem, where each customer location can be visited multiple times. In this problem, the total demand for multiple products of a customer can be satisfied by multiple deliveries by one or more vehicles, while the split delivery of a product is not allowed.

For the one-to-many-to-one PDP class, Gribkovskaia et al. [14] proposed tabu search (TS) to solve the SVRPDSP. TS could generate a near-optimal solution to a number of tested instances from the VRPLIB. Tasan and Gen [5] proposed a genetic algorithm to solve the VRPSPD. The computation result based on medium-sized instances showed that the best solutions from GA performed better than the upper bound obtained from CPLEX. Goksal et al. [6] proposed a heuristic based on particle swarm optimization (PSO) with variable neighborhood descent (VND) local search to solve the VRPSPD. The algorithm can successfully improve some best-known solutions of the standard problem set. The overall performance is comparable with other effective heuristics. Avci and Topaloglu [7] proposed an adaptive local search algorithm that is a hybrid of simulated annealing and variable neighborhood search. The developed adaptive-threshold function enables the algorithm parameters to be self-tuned. The algorithm was used to solve both the VRPSPD and VRPMPD. Computational results demonstrated the algorithm effectiveness in solving the problems within reasonable computation times. Later, Avci and Topaloglu [9] proposed an adaptive hybrid local search (HSL) that integrates adaptive-threshold acceptance with tabu search to solve the heterogeneous VRPSPD. For the VRPDDP, proposed by [15], an efficient cooperative variable neighborhood search (CVNS) was constructed to solve the problem. The algorithm can successfully improve best-known solutions of the available instances of the problem. Finally, Drexl [30] developed branch-and-cut algorithms for solving the VRPTT. The algorithms are tested with problem instances that are designed to resemble real-world VRPTT.

Recent algorithm development for solving a one-to-one PDP and its extensions is as follows. Qu and Bard [18] proposed a greedy randomized adaptive search procedure (GRASP) with adaptive large neighborhood search for pickup and delivery with transshipment (PDPT). The problem under study allows aircraft to transfer a load to another aircraft at some location (a transshipment) and then identify situations where cost savings can be gained from the transshipment. Parragh and Schmid [19] proposed a hybrid column generation and large neighborhood search for a DARP that minimized the total routing costs under the restrictions of maximum user ride time, maximum trip time, vehicle capacity, and time window. Kirchler and Wolfler Calvo [20] proposed a granular tabu search algorithm that features a granular (or reduced) neighborhood, which only contains moves that potentially lead to good solutions, to solve the DARP. The proposed tabu search performance, tested on standard instances, is comparable with other existing algorithms, i.e., TS, GA, and VNS. Masson et al. [21] developed an adaptive large neighborhood search (ALSN) to solve a dial-a-ride problem 
with transfer (DARPT). A transfer point, where users can change vehicles, is introduced into DARP to reduce the transportation cost. Experimental results indicated that adding a transfer point could decrease the transportation cost by $8 \%$. Rais et al. [22] proposed a new mixed integer programming model for the PDPT. The transshipment in this problem is referred to as the transferring of loads between vehicles at some designated transshipment locations. The model is generalized to handle heterogeneous vehicles and multiple origin and final destination nodes. It should be noted that the transshipment feature in the DARPT is for the transferring of loads between vehicles. This is different from the transshipment considered in this paper, which refers to the transferring of items between nodes of customers. Finally, Mues and Pickl [25] proposed a column generation technique to solve the PDPTTW in two numerical examples.

\section{Mathematical Model}

3.1. Problem Description. The VRPT is defined as follows:

(1) The network has a single depot.

(2) There are multiple customer nodes, each of which has a regular demand and may request a transshipment demand.

(3) Each customer node must be visited once by a vehicle.

(4) Each route must start and end at the depot.

(5) The total load of a vehicle must not exceed its capacity.

(6) Each vehicle has the same capacity.

(7) The total transportation distance is to be minimized.

(8) The pickup operation must occur before the delivery operation for a transshipment demand, i.e., the pickup node must be visited before the delivery node of the transshipment demand.

(9) At any particular pickup or delivery node, the transshipment demand is always smaller than the regular demand. This indicates that there is no need to explicitly consider the load of the transshipment demand. That is, at the pickup node, the regular demand is unloaded for delivery first, which always leaves enough space on the truck for the transshipment demand to be picked up.

(10) Although it is very rare, in the case that there are two conflicting transshipment demand requests, one from node $i$ to node $j$ and the other from node $j$ to node $i$, only one of the two transshipment demands may be satisfied on the same route. For example, if the transshipment demand from $i$ to $j$ is chosen, then the item of the transshipment demand from $j$ to $i$ will be picked up when the vehicle visits node $j$ and brought back to the depot, and then the item will be delivered to node $i$ on the next delivery day.
3.2. Mathematical Formulation. The mathematical model that represents VRPT is formulated as a mixed integer linear programming (MILP) model. The model is an extension of the model in [5]. The model uses the following notation.

$I$ : set of demand nodes. $I=\{1,2, \ldots, n\}$.

$I_{0}$ : set of all nodes including depot.

$I_{0}=\{0,1,2, \ldots, n\}$. $O$ : set of depots. $O=\{0\}$.

$V$ : set of vehicles. $V=\{1,2, \ldots, m\}$.

$P D_{i j}$ : set of transshipment demand from nodes $i$ to $j$.

\subsubsection{Parameters}

C: vehicle capacity.

$d_{i j}$ : transportation distance associated with traveling from nodes $i$ to $j$, where $i, j \in I_{0}, i \neq j$.

$D_{i}$ : amount of regular demand at node $i \in I$.

$n$ : number of nodes including the depot.

\subsubsection{Decision Variables}

$l_{v}$ : load of vehicle when leaving the depot.

$S_{i}$ : variable used to avoid subtours, interpreted as the position of node $i \in I$ in the route.

$x_{i j v}$ : binary decision variable that indicates whether the $v$ th vehicle travels from nodes $i$ to $j$.

$s e_{i v}$ : variable used to indicate the sequence of demand node $i$ in vehicle $v$.

\subsubsection{Objective Function.}

$$
\min \sum_{i \in I_{0}} \sum_{j \in I_{0}} \sum_{v \in V} d_{i j} x_{i j v}
$$

subject to

$$
\begin{gathered}
\sum_{i \in I_{0}} \sum_{v \in V} x_{i j v}=1, \quad \forall j \in I, \\
\sum_{i \in I_{0}} x_{i k v}=\sum_{j \in I_{0}} x_{k j v}, \quad \forall k \in I, \forall v \in V \\
\sum_{i \in O} \sum_{j \in I} x_{i k v} \leq 1, \quad \forall v \in V, \\
\sum_{i \in I_{0}} \sum_{j \in I_{0}} D_{i} x_{i j v} \leq C, \quad \forall v \in V, \\
S_{j}-S_{i} \geq 1-\left(n\left(1-\sum_{v \in V} x_{i j v}\right)\right), \quad \forall i \in I, \forall j \in I, i \neq j, \\
s e_{i v}+(n+1) x_{i j v} \leq s e_{j v}+n, \quad \forall i \in I, \forall j \in I, \forall v \in V, i \neq j,
\end{gathered}
$$




$$
\begin{aligned}
s e_{i v} & \leq s e_{j v}, \quad \forall(i, j) \in P D_{i j}, \forall v \in V, \\
s e_{j v} & \geq \sum_{i \in I_{0}} x_{i j v}, \quad \forall j \in I, \forall v \in V, \\
s e_{j v} & \leq n \sum_{i \in I_{0}} x_{i j v}, \quad \forall j \in I, \forall v \in V, \\
x_{i k v} & \in\{0,1\}, \quad \forall i \in I, \forall j \in I, \forall v \in V, \\
l_{v} & \geq 0, \quad \forall v \in V, \\
S_{i} & \geq 0, \quad \forall i \in I, \\
s e_{i v} & \geq 0, \quad \forall i \in I, \forall v \in V .
\end{aligned}
$$

The objective function (1) is to minimize the total transportation distance by all vehicles. Constraint (2) ensures that each customer is visited once by one vehicle only. Constraint (3) ensures that if a vehicle enters a node, it must leave the node. Constraint (4) ensures that each vehicle does not leave the depot more than once. Constraint (5) limits the load that each vehicle can carry. Constraint (6) is used to prevent subtours.

Additional constraints that incorporate transshipment demands are constraints (7)-(10). Constraint (7) specifies the sequence of nodes that are visited by a vehicle. That is, if a vehicle $v$ travels from node $i$ to node $j$, then $x_{i j v}=1$. Constraint (7) becomes $s e_{i}+1 \leq s e_{j}$, which forces $s e_{i}$ to be less than $s e_{j}$. In other words, if node $i$ is visited before node $j$, then their sequences on the route must be consistent. However, if vehicle $v$ does not travel from node $i$ to node $j$, then there is no restriction on $s e_{i}$ and $s e_{j}$. Constraint (8) ensures that for each pair of nodes of a transshipment demand, the pickup node must be visited before the delivery node (by the same vehicle). Constraints (9) and (10) specify that the lower bound and upper bound of the sequence of nodes visited by a vehicle are 1 and $n$, respectively. Finally, constraints (11)-(14) specify the types of decision variables.

\section{Model Testing on Small Problem Instances: Optimal Solution Results and Analysis}

To demonstrate MILP model for the VRPT, we develop a new problem instance set, called set $T$ in this paper, which consists of six small problem instances. These problem instances are generated such that they resemble the real problem faced by the retail chain where the VRPT originates. Each instance has a total of 20 nodes, including a depot and 19 customers (retail stores). Each store has a daily regular demand. Among the stores, there are four transshipment demand requests, denoted as T1, T2, T3, and T4. Each transshipment demand is a pair of pickup and delivery customers. An example of small problem instance with actual store locations is illustrated in Figure 2. There are two homogenous trucks to satisfy delivery demands. The list of the instances is shown in Table 2.

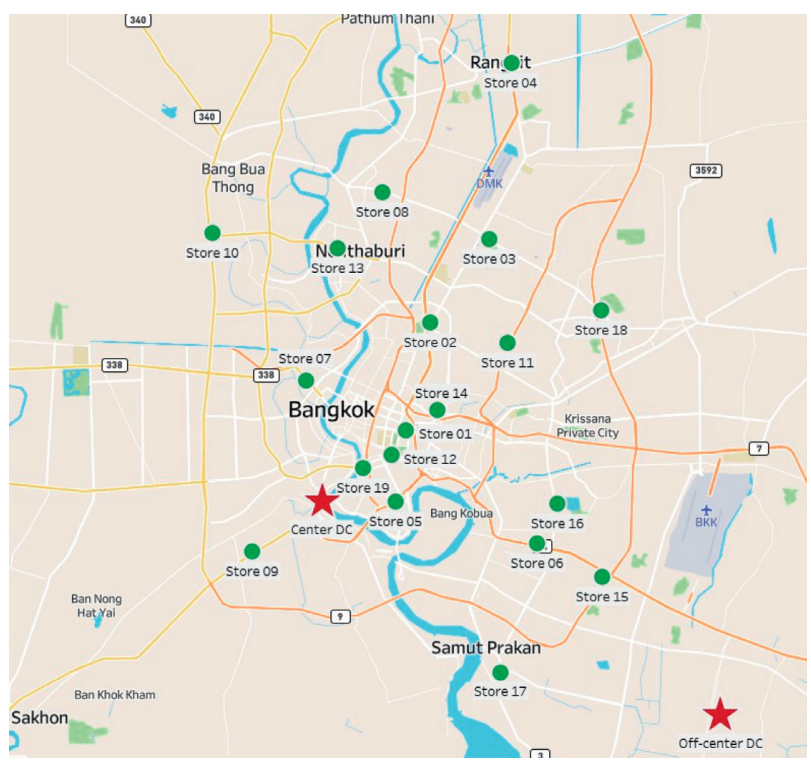

FIgURE 2: Example of a small problem instance.

TABLe 2: List of set $\mathrm{T}$ instances.

\begin{tabular}{lccc}
\hline Instance code & Truck cap. & Total demand & D/C ratio \\
\hline CEN T2G & 140 & 266 & 0.95 \\
CEN T2H & 140 & 252 & 0.90 \\
CEN T2J & 140 & 238 & 0.85 \\
OFF T2G & 140 & 266 & 0.95 \\
OFF T2H & 140 & 252 & 0.90 \\
OFF T2J & 140 & 238 & 0.85 \\
\hline
\end{tabular}

Note. $\mathrm{D} / \mathrm{C}$ ratio is the ratio between total demand and total truck capacity.

From the list of small problem instances in Table 2, CEN represents the instances where the locations of the depot are relatively at the center and the locations of customers are scattered randomly around the depot. OFF represents the instances where the locations of the depot are far away from the locations of all the customer locations. The instances of CEN and OFF with the same codes (i.e., 2TG, 2TH, and 2TJ) indicate that the store locations, demands, and pickup and delivery stores of the transshipment demands are identical.

Since the motivation of this problem is from a real problem, the small problem instances include two locations of the depot. One depot, currently in use by the retail chain, is represented as the CEN location, which is located in the middle of the city, where the current capacity utilization is almost $100 \%$ and expansion is no longer possible. The other depot is at the OFF location, which represents the future location of a new larger depot, around $20 \mathrm{~km}$ from the city. The 19 customer locations are generated to represent the department stores with high demand.

To analyze the changes in the objective function from satisfying one or more of these transshipment demands, each instance is solved 16 times, one time as a VRP and 15 times as VRPTs, with the parameters in Table 3. The VRP (Run 1) instance is solved by excluding all transshipment demands. The 15 VRPTs (Runs 2-16) are all possible combinations that include at least one of the four 
TABle 3: Optimal total costs for VRP (Run 1) and VRPT (Runs 2-16) and percentage difference between VRPT and VRP total costs.

\begin{tabular}{|c|c|c|c|c|c|c|c|c|c|c|c|c|c|c|c|c|}
\hline \multirow{2}{*}{ Run } & \multicolumn{4}{|c|}{ Trans. demand } & \multicolumn{2}{|c|}{ CEN T2G } & \multicolumn{2}{|c|}{ CEN T2H } & \multicolumn{2}{|c|}{ CEN T2J } & \multicolumn{2}{|c|}{ OFF T2G } & \multicolumn{2}{|c|}{ OFF T2H } & \multicolumn{2}{|r|}{ OFF T2J } \\
\hline & $\mathrm{T} 1$ & $\mathrm{~T} 2$ & $\mathrm{~T} 3$ & $\mathrm{~T} 4$ & Opt. & $\%$ difference & Opt. & $\%$ difference & Opt. & $\%$ difference & Opt. & $\%$ difference & Opt. & $\%$ difference & Opt. & $\%$ difference \\
\hline 1 & & & & & 429 & & 437 & & 408 & & 407 & & 423 & & 446 & \\
\hline 2 & $\mathrm{x}$ & & & & 433 & 0.93 & 437 & 0.00 & 429 & 5.15 & 421 & 3.44 & 423 & 0.00 & 446 & 0.00 \\
\hline 3 & & $\mathrm{x}$ & & & 433 & 0.93 & 445 & 1.83 & 418 & 2.45 & 411 & 0.98 & 460 & 8.75 & 459 & 2.91 \\
\hline 4 & & & $\mathrm{x}$ & & 433 & 0.93 & 462 & 5.72 & 426 & 4.41 & 446 & 9.58 & 450 & 6.38 & 446 & 0.00 \\
\hline 5 & & & & $\mathrm{x}$ & 459 & 6.99 & 447 & 2.29 & 408 & 0.00 & 443 & 8.85 & 453 & 7.09 & 471 & 5.61 \\
\hline 6 & $\mathrm{x}$ & $\mathrm{x}$ & & & 433 & 0.93 & 473 & 8.24 & 440 & 7.84 & 421 & 3.44 & 465 & 9.93 & 469 & 5.16 \\
\hline 7 & $\mathrm{x}$ & & $\mathrm{x}$ & & 433 & 0.93 & 492 & 12.59 & 429 & 5.15 & 447 & 9.83 & 456 & 7.80 & 446 & 0.00 \\
\hline 8 & $\mathrm{x}$ & & $\mathrm{x}$ & & 459 & 6.99 & 468 & 7.09 & 440 & 7.84 & 476 & 16.95 & 453 & 7.09 & 489 & 9.64 \\
\hline 9 & & $\mathrm{x}$ & $\mathrm{x}$ & & 433 & 0.93 & 475 & 8.70 & 434 & 6.37 & 458 & 12.53 & 479 & 13.24 & 459 & 2.91 \\
\hline 10 & & $\mathrm{x}$ & & $\mathrm{X}$ & 473 & 10.26 & 483 & 10.53 & 418 & 2.45 & 464 & 14.00 & 474 & 12.06 & 476 & 6.73 \\
\hline 11 & & & $\mathrm{x}$ & $\mathrm{x}$ & 513 & 19.58 & 477 & 9.15 & 455 & 11.52 & 486 & 19.41 & 475 & 12.29 & 474 & 6.28 \\
\hline 12 & $\mathrm{X}$ & $\mathrm{x}$ & $\mathrm{X}$ & & 433 & 0.93 & 523 & 19.68 & 454 & 11.27 & 458 & 12.53 & 491 & 16.08 & 478 & 7.17 \\
\hline 13 & $\mathrm{x}$ & $\mathrm{x}$ & & $\mathrm{x}$ & 480 & 11.89 & 504 & 15.33 & 440 & 7.84 & 476 & 16.95 & 474 & 12.06 & 501 & 12.33 \\
\hline 14 & $\mathrm{X}$ & & $\mathrm{x}$ & $\mathrm{x}$ & 514 & 19.81 & 502 & 14.87 & 482 & 18.14 & 489 & 20.15 & 489 & 15.60 & 494 & 10.76 \\
\hline 15 & & $\mathrm{x}$ & $\mathrm{x}$ & $\mathrm{x}$ & 513 & 19.58 & 509 & 16.48 & 455 & 11.52 & 493 & 21.13 & 500 & 18.20 & 476 & 6.73 \\
\hline 16 & $\mathrm{X}$ & $\mathrm{x}$ & $\mathrm{x}$ & $\mathrm{x}$ & 514 & 19.81 & 532 & 21.74 & 483 & 18.38 & 501 & 23.10 & 500 & 18.20 & 501 & 12.33 \\
\hline
\end{tabular}

transshipment demands that must be satisfied. For examples, Run 8 includes two transshipment demands (T1 and T3), and Run 16 includes all four transshipment demands. The instances are solved with CPLEX solver. The results from all instances are also shown in Table 3, including the optimal total distance and the percentage difference in the optimal total distance between each VRPT instance and VRP instance.

The results demonstrate that small problem instances of VRPT can be solved optimally by the proposed MILP. The optimal total transportation distance increases as more transshipment demands are satisfied. An important insight learned is that there are pairs of transshipment demands that are relatively in conflict with each other, such that including these transshipment demands together on the same route results in a significant increase in the total transportation distance. These are demand pairs where their respective direct paths (from the pickup and delivery nodes) intersect with each other. The results of the runs that include one or more of these pairs are highlighted in bold in Table 3. By eliminating one of the transshipment demands whose direct paths intersect, the total transportation distance is likely to be reduced significantly. This observation leads to the development of a mechanism that helps to choose the transshipment demands that should not be included together on the same route, given that satisfying a transshipment demand is optional.

\section{Hybrid Threshold Accepting and Neighborhood Search Heuristics}

To solve medium to large instances of VRPT, we develop two heuristic algorithms for the VRPT, where all transshipment demands are enforced and where partial transshipment demands are satisfied (the problem becomes a so-called VRP with optional transshipment or VRPOT). Both algorithms are a hybrid of the threshold accepting (TA) algorithm and neighborhood search (NS). The optional transshipment version adds a predetermined forbidden list that contains pairs of transshipment demands that are not allowed to be satisfied on the same route. The algorithm with forbidden list can then generate a good solution that delivers a partial of transshipment demands without incurring too much additional delivery distance. The hybrid algorithms are shown in Figure 3.

The algorithm description is based on the following notation and definitions.

New solution: a solution generated by neighborhood search.

Current solution: a solution that is used in the comparison.

Best solution: solution with the best-so-far value.

Tmax: the highest level of threshold.

Tcur: the current threshold value.

Tmin: the minimum threshold value.

$\alpha$ : threshold reduction parameter.

The algorithm consists of five main components. The pseudocode of TA-NS is shown in Figure 4. The function and description of each component are described as follows.

5.1. Component 1: Generating Initial Solution. The initial solution has a significant impact on the algorithm performance. A good initial solution may help to reduce the number of iterations required to obtain a good quality final solution. In this component, we use four methods to generate an initial solution. All four methods to generate initial solutions are used when solving each problem instance. This 


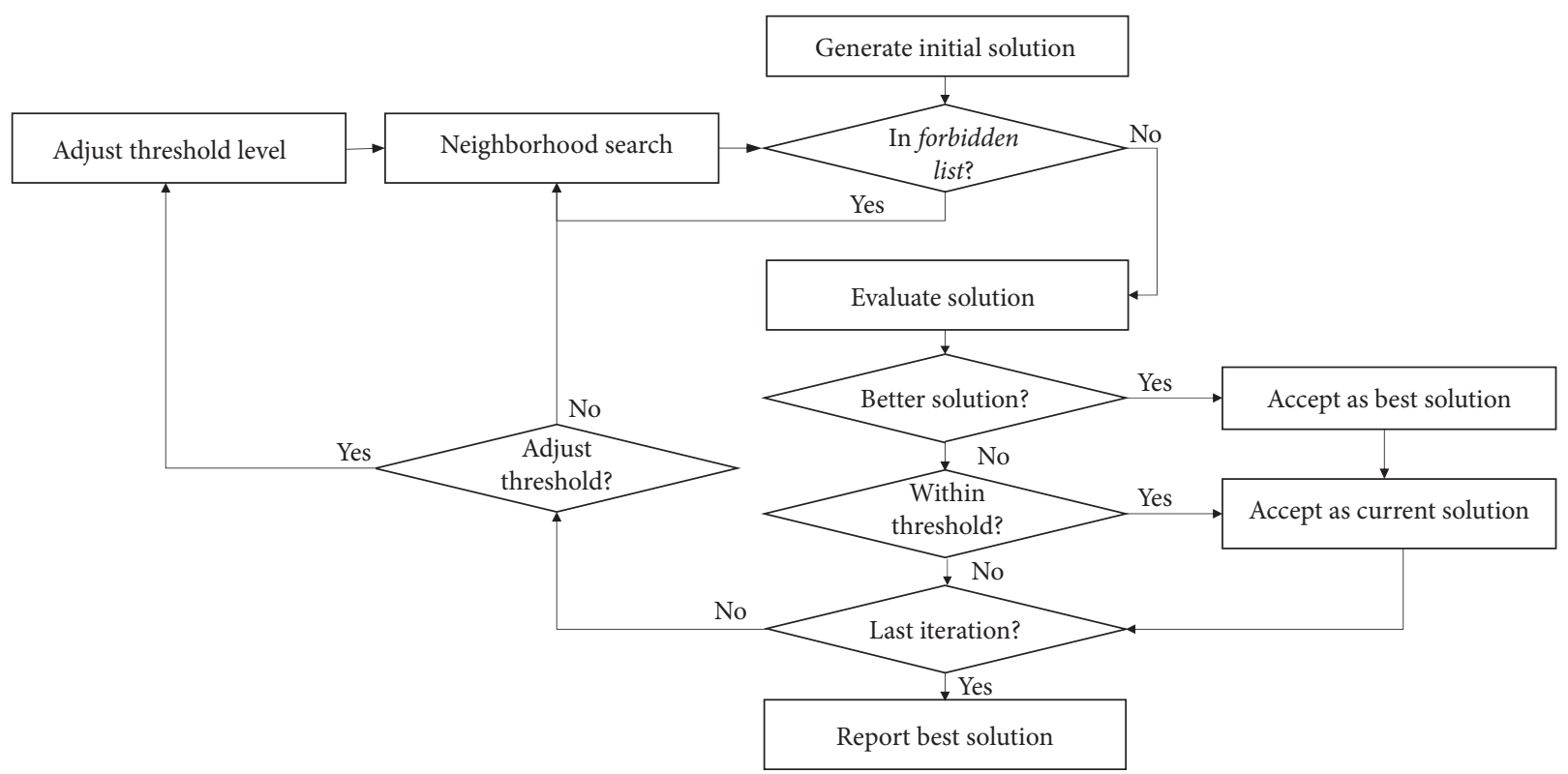

FIgURE 3: A TA-NS algorithm diagram.

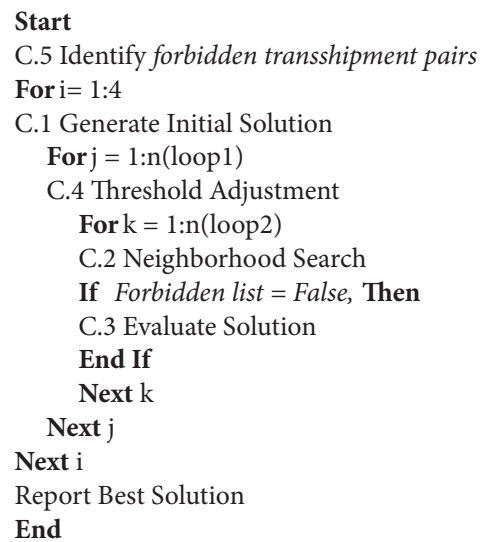

Figure 4: Pseudo-code of the TA-NS algorithm.

is because, based on initial performance testing, none of the methods can consistently outperform the other methods.

\subsubsection{Random Generation. Initial solution is randomly generated.}

5.1.2. Sweep Algorithm. The standard sweep algorithm [38] proceeds as follows. The algorithm chooses a node as the starting point of a route and looks for the closest node to visit. Note that the closest node in the sweep algorithm is the closest node from sweeping of a clock hand, rather than in terms of the closest distance. A clockwise sweep looks for the closest node on the right of the current node, while counterclockwise looks for the closest node on the left side. The algorithm is performed in both directions, clockwise and counter-clockwise. In each direction, each customer node alternates as the starting point of the sweep. The best sweep solution is then used as the initial solution to the TA-NS algorithm.

5.1.3. Savings Algorithm. This is a well-known heuristic for VRP developed by Clarke and Wright [39]. The algorithm starts by generated one round trip from the depot to each demand node. Then, the algorithm proceeds to combine two routes with the most distance (or cost) savings first and repeatedly combines the routes with the next best savings, until the final routes are generated (no more combining is possible). This method can provide a good solution for VRP, but may not be a good solution for VRPT since the method does not consider the pickup and delivery requirements.

5.1.4. Pickup-Delivery Heuristics. We develop a heuristic to generate a good initial solution for VRPT. This heuristic proceeds as follows. First, the VRPT is solved using TA-NS without considering the transshipment demand, i.e., solving a VRP. With the VRP solution as an initial solution, the heuristic adds one randomly chosen transshipment demand at a time. Suppose for a particular transshipment demand, the pickup node and delivery nodes are on different routes in the VRP solution. A good pickup and delivery is generated by deletion of a pickup (or delivery) node from its route and insertion to the other route that contains its corresponding delivery (or pickup) node so that they are on the same route. Choosing the order at which the transshipment demands are satisfied, the nodes (pickup or delivery) to delete, as well as the insertion point, is performed by choosing the one that results in relatively little change in the total route distance 
first. After satisfying the added transshipment demand, the heuristic proceeds to add the next transshipment demand.

5.2. Component 2: Neighborhood Search. Neighborhood search is an important mechanism to generate a new solution in this algorithm. We use four methods for NS: swapping, inserting, 2-opt, and grouping.

Swapping: randomly swap the positions between two selected nodes.

Inserting: randomly eliminate one node and insert it elsewhere.

2-opt: randomly select two arcs and swap the node pairs of those arcs.

Grouping: randomly cluster nodes into groups of adjacent nodes and then swap the position of the groups.

These methods are randomly applied to the current solution to obtain the new solution.

5.3. Component 3: Evaluating the Solution. To evaluate a solution, we define the objective function and accepting mechanism as follows.

5.3.1. Objective Function. To solve the VRPT, we modify the objective function that originally contains only the total distance, which does not consider whether or not the transshipment demand(s) are satisfied. The new objective function includes a penalty for a solution that does not satisfy the transshipment demand. This increases the chance for the algorithm to discover better solutions that satisfy transshipment demands with lower distances. Let $p$ be the percentage of transshipment demand satisfaction. The new objective function is defined as follows:

objective function $= \begin{cases}\text { total distance } *(1+(k-p)), & p<1, \\ \text { total distance, } & p=1 .\end{cases}$

From equation (15), if all transshipment demands are satisfied, then the objective function is simply to minimize the total distance. However, if a percentage $p$ of all transshipment demands is satisfied, then the objective function is penalized by a multiplication factor of $1+(k-p)$. Based on our extensive tests, $k=1.1$ is effective for the algorithm to find good solutions that properly choose transshipment demands to be satisfied. In addition, by using equation (15) as the objective function, the algorithm becomes effective in diversifying its search space. In other words, given a solution and the mechanism of TA and NS, the penalty function can make the algorithm jump to a solution space that cannot be previously reached.
5.3.2. Accepting Mechanism. After evaluating a new solution, it is compared with the best solution and the current solution. Three scenarios can occur: (1) accepting as best solution, if the new solution is better than the previous best solution; (2) accepting as current solution, if the new solution satisfies the condition (new solution-current solution) $\leq$ Tcur, which allows the new solution to have a worse objective function value than the current solution by the value of Tcur; or (3) rejecting the new solution.

5.4. Component 4: Threshold Adjustment. Adjusting the threshold level plays an important role since an improper threshold level increases the computational time and may lead to low-quality results. The threshold value begins with the Tmax value. Then, it is reduced by a fractional factor $\alpha$, i.e., $T c u r=T_{c u r}^{*} \alpha$ when the iteration number reaches a specified value. The adjustment process repeats until the threshold value reaches Tmin.

5.5. Component 5: Identifying Forbidden Transshipment Pairs. This component is applied only when the objective is to partially satisfy the transshipment demand, i.e., the VRPOT. The purpose is to avoid solutions that include pairs of transshipment demands that may significantly increase the transportation distance. A procedure is developed to identify a list of forbidden transshipment pairs before solving the problem. An example of forbidden transshipment pairs is shown in Figure 5. The steps are described as follows:

(1) For a given pair of transshipment demands (from nodes 3 to 5 and from nodes 7 to 16 in Figure 5), determine the intersection point between two straight lines that connect the pickup and delivery nodes of the pair of transshipment demands.

(2) If the two lines intersect (i.e., the two lines form a crossed path in the route), then compute the total distance of the two lines.

(3) Compute the border length of the map. In Figure 5, the border length is the total distance from node 15 $\longrightarrow$ node $17 \longrightarrow$ node $9 \longrightarrow$ node $10 \longrightarrow$ node 8 $\longrightarrow$ node $3 \longrightarrow$ node $18 \longrightarrow$ node 15 . If the total distance of the two lines is greater than a factor of $25 \%$ of the border length, then the transshipment pair is considered to be forbidden. Note that an extensive testing is conducted to fine-tune the value of this factor.

After all possible pairs of transshipment demands are evaluated to obtain a list of forbidden transshipment pairs, the TA-NS algorithm proceeds to search for a new solution as if all transshipment demands will be satisfied. After a new solution is created, if it contains one or more pairs of transshipment demands in the forbidden list, then the 


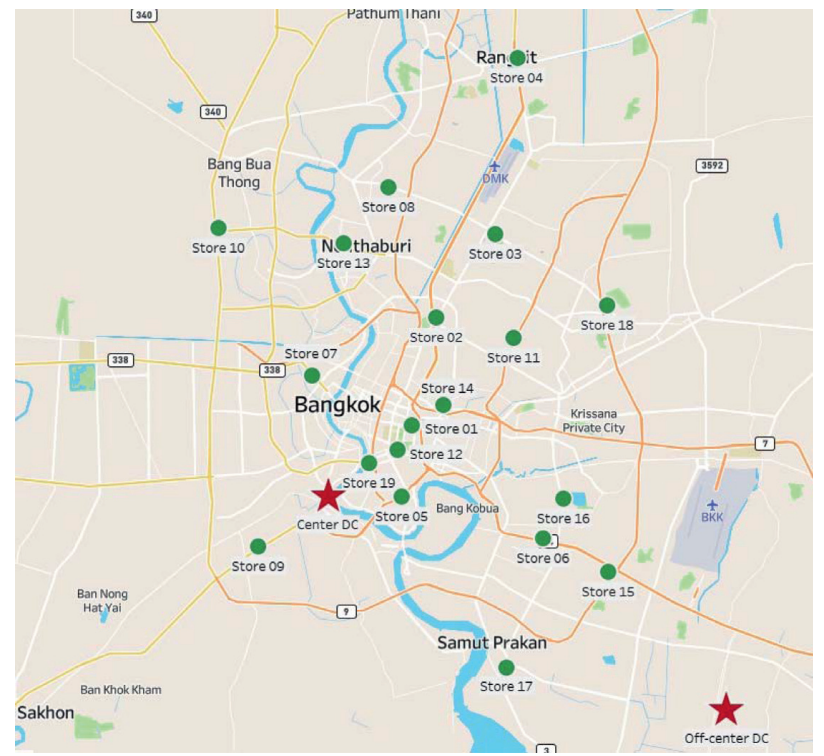

FIGURE 5: Example of forbidden transshipment pairs.

solution is discarded without being evaluated. As a result, an accepted solution is restricted to satisfy up to only one transshipment demand in each forbidden transshipment pair, but not both.

\section{Computational Experiments}

6.1. Problem Instances. The performance of TA-NS is evaluated by using two problem instance sets. First, the TA-NS is tested on the small problem instance Set T, which contains 20-node instances with $20 \%$ transshipment demands with respect to the number of nodes, as in the previous numerical example. Second, for the medium to large problem instances, we adapt the standard problem instances, set A [40]. This standard problem set is selected because the problem instances are very similar to the current network of the industrial user of the study, i.e., the DC is in the city with retail stores geographically scattered around the DC. Then, the transshipment demands were randomly generated and added to the standard problem instances. To make the performance evaluation consistent with that of the small problem instances, the number of transshipment demands is also set to $20 \%$ of the total number of nodes of each instance of set A. In the computation test, TA-NS is applied to solve the VRPT (i.e., all transshipment demands are enforced) and VRPOT (with the forbidden list added to TA-NS).

6.2. Small Problem Instance Results. Table 4 shows the optimal solution of Run 16 of the VRPT (i.e., all four transshipment demands must be satisfied), the best-found solutions, the off-optimal percentage of the VRPT solved by TA-NS heuristic, and VRPOT solved by TA-NS heuristic with the forbidden list. From the VRPT results, TANS can obtain optimal solutions in two out of six instances, with an average percent off-optimal of $0.82 \%$ ( $0.51 \%$ for CEN instances and $1.13 \%$ for OFF instances). In addition, from the VRPOT results, the TA-NS heuristic with forbidden list can find optimal solution in three out of six instances, with an average off-optimal percentage of $1.82 \%(0.61 \%$ for CEN instances and $3.03 \%$ for OFF instances).

6.3. Large Problem Instance Results. Table 5 shows the offoptimal percentage for the optimal solutions of the VRP and TA-NS best solutions, for both the VRPT and VRPOT. For performance evaluation, we use the optimal solution of the VRP as the lower bound of the VRPT.

From the table, the best-found CVPRT solutions from the TA-NS algorithm are, on average, $26.44 \%$ higher than those of the lower bound. This is to be expected, given that the optimal solutions of the VRPT for these instances are unknown, and that the lower bounds are likely to be much lower than the optimal solution of the VRPT. It is important to emphasize that, based on the 20 -node small problem instances (set T) where the optimal solutions of the VRPT are found, the average percent difference for the lower bound (Run 1 or the first row of Table 3) and the optimal solution of the VRPT is $18.93 \%$ (Run 16 or the last row of Table 3 ). This implies that the best-found solutions of the VRPT from the TA-NS algorithm should be much closer to the optimal VRPT than to the lower bound, which suggests that the TA-NS algorithm is effective.

For the VRPOT, on average, 67\% of the transshipment demands are satisfied with an average percent difference of $13.75 \%$ between the best-found VRPOT solution and the lower bound. Moreover, the best-found solutions of the VRPOT are approximately 50\% closer to the lower bound, when compared with the best-found solution of the VRPT (i.e., $13.75 \%$ vs. $26.44 \%$ ). The results clearly demonstrate the effectiveness of TA-NS with the forbidden list in finding 
TABLE 4: Solution comparison for Run 16 of set T instance.

\begin{tabular}{|c|c|c|c|c|c|c|c|}
\hline \multirow{2}{*}{ Problem instance } & \multicolumn{3}{|c|}{ VRPT (Run 16) } & \multicolumn{4}{|c|}{ VRPOT (Run 16) } \\
\hline & Opt. & Best found & $\%$ OFF-OPT. & Opt. & Best found & TD satisfied & $\%$ OFF-OPT. \\
\hline CEN T2G & 514 & 514 & 0.00 & 433 & 433 & T1, T2, T3 & 0.00 \\
\hline CEN T2H & 532 & 539 & 1.32 & 437 & 445 & $\mathrm{~T} 1$ & 1.83 \\
\hline CEN T2J & 483 & 484 & 0.21 & 440 & 440 & $\mathrm{~T} 1, \mathrm{~T} 2, \mathrm{~T} 4$ & 0.00 \\
\hline OFF T2G & 501 & 501 & 0.00 & 458 & 458 & $\mathrm{~T} 1, \mathrm{~T} 2, \mathrm{~T} 3$ & 0.00 \\
\hline $\mathrm{OFF} \mathrm{T} 2 \mathrm{H}$ & 500 & 507 & 1.40 & 423 & 450 & T3 & 6.38 \\
\hline OFF T2J & 501 & 511 & 2.00 & 478 & 491 & $\mathrm{~T} 1, \mathrm{~T} 2, \mathrm{~T} 3$ & 2.72 \\
\hline
\end{tabular}

TABle 5: Comparison between the best-found solutions from TA-NS and the lower bound (optimal solution of VRP) of set A.

\begin{tabular}{|c|c|c|c|c|c|c|c|c|}
\hline $\begin{array}{l}\text { Problem } \\
\text { instance }\end{array}$ & $\begin{array}{c}\text { Lower bound } \\
\text { (optimal CVRP) }\end{array}$ & $\begin{array}{c}\text { No. of } \\
\text { TD }\end{array}$ & $\begin{array}{c}\text { Upper bound } \\
\text { CVRPT }\end{array}$ & $\begin{array}{l}\text { Best Sol. } \\
\text { CVRPT }\end{array}$ & $\begin{array}{c}\% \text { Diff from } \\
\text { lower bound (\%) }\end{array}$ & $\begin{array}{l}\text { Best sol. } \\
\text { CVRPOT }\end{array}$ & $\begin{array}{l}\text { \# of TD } \\
\text { satisfied }\end{array}$ & $\begin{array}{c}\% \text { Diff from } \\
\text { lower bound (\%) }\end{array}$ \\
\hline A n $32 \mathrm{k} 5$ & 784 & 6 & 1065 & 934 & 19.13 & 862 & 3 & 9.95 \\
\hline A n $33 \mathrm{k} 5$ & 661 & 6 & 807 & 775 & 17.25 & 762 & 5 & 15.28 \\
\hline A n33 k6 & 742 & 6 & 862 & 847 & 14.15 & 809 & 5 & 9.03 \\
\hline A n $34 \mathrm{k} 5$ & 778 & 7 & 1015 & 888 & 14.14 & 888 & 7 & 14.14 \\
\hline A n 36 k5 & 799 & 7 & 1138 & 1019 & 27.53 & 928 & 6 & 16.15 \\
\hline A n $37 \mathrm{k} 5$ & 669 & 7 & - & 839 & 25.41 & 743 & 4 & 11.06 \\
\hline A n $37 \mathrm{k} 6$ & 949 & 7 & 1395 & 1141 & 20.23 & 989 & 4 & 4.21 \\
\hline A n $38 \mathrm{k} 5$ & 730 & 8 & - & 948 & 29.86 & 847 & 7 & 16.03 \\
\hline A n $39 \mathrm{k} 5$ & 822 & 8 & - & 935 & 13.75 & 909 & 7 & 10.58 \\
\hline A n39 k6 & 831 & 8 & 1117 & 1055 & 26.96 & 978 & 7 & 17.69 \\
\hline A n $44 \mathrm{k} 6$ & 937 & 9 & - & 1175 & 25.40 & 1104 & 7 & 17.82 \\
\hline A n $45 \mathrm{k} 6$ & 944 & 9 & - & 1243 & 31.67 & 1073 & 5 & 13.67 \\
\hline A n $45 \mathrm{k} 7$ & 1146 & 9 & - & 1354 & 18.15 & 1331 & 7 & 16.14 \\
\hline A n $46 \mathrm{k} 7$ & 914 & 9 & - & 1139 & 24.62 & 1013 & 6 & 10.83 \\
\hline A n $48 \mathrm{k} 7$ & 1073 & 10 & - & 1490 & 38.86 & 1216 & 6 & 13.33 \\
\hline A n53 k7 & 1010 & 11 & - & 1402 & 38.81 & 1188 & 6 & 17.62 \\
\hline A n54 k7 & 1167 & 11 & - & 1446 & 23.91 & 1336 & 7 & 14.48 \\
\hline A n $55 \mathrm{k} 9$ & 1073 & 11 & - & 1350 & 25.82 & 1203 & 7 & 12.12 \\
\hline A n60 k9 & 1354 & 12 & - & 1790 & 32.20 & 1495 & 5 & 10.41 \\
\hline A n61 k9 & 1035 & 12 & - & 1297 & 25.31 & 1194 & 8 & 15.36 \\
\hline A n62 k8 & 1288 & 12 & - & 1744 & 35.40 & 1484 & 7 & 15.22 \\
\hline A n63 k9 & 1634 & 13 & - & 1983 & 21.36 & 1851 & 7 & 13.28 \\
\hline A n63 k10 & 1315 & 13 & - & 1749 & 33.00 & 1518 & 6 & 15.44 \\
\hline A n64 k9 & 1402 & 13 & - & 1802 & 28.53 & 1558 & 7 & 11.13 \\
\hline A n65 k9 & 1177 & 13 & - & 1647 & 39.93 & 1378 & 9 & 17.08 \\
\hline A n69 k9 & 1159 & 14 & - & 1588 & 37.01 & 1357 & 9 & 17.08 \\
\hline A n 80 k10 & 1764 & 16 & - & 2214 & 25.51 & 2047 & 10 & 16.04 \\
\hline
\end{tabular}

good delivery routes which satisfy only the transshipment demands that would not incur much additional distance.

Among all instances of set A, there are only seven instances where the upper bound can be obtained by solving the VRPT using CPLEX solver for 3,600 seconds. A comparison of the results for these seven instances is shown in Figure 6. For these instances, the best-found solution of the VRPT is better than the upper bound obtained by CPLEX solver by an average of $9.25 \%$.

Finally, Table 6 shows the average percentage of the additional distance (from the lower bound) per each satisfied transshipment demand, for the best-found VRPT solutions and the best-found VRPOT solutions. The percentage of the additional distance per satisfied transshipment demand is a fair index to evaluate the performance of the forbidden transshipment pairs. From the results, 20 out of 27 instances have lower percentages of the additional distance per satisfied transshipment demand with the use of a forbidden list of transshipment pairs. The average percentage of the additional distance per transshipment in the VRPT and VRPOT is $2.75 \%$ and $2.19 \%$, respectively, with an average of $0.56 \%$ improvement from implementing the forbidden list of transshipment pairs.

6.4. Discussion. The computational test results on the small problem instance set $\mathrm{T}$ and large problem instance set $\mathrm{A}$ indicate effectiveness of the proposed TA-NS heuristic for VRPT and forbidden list for VRPOT. In the real problem, there are two business units (BUs) of the retail chain consisting of 22 store locations in Bangkok. The depot makes delivery service to 15 high demand locations out of 22 locations on a daily basis, except on Sunday, and to 7 medium demand locations on alternate day basis. In other words, the 


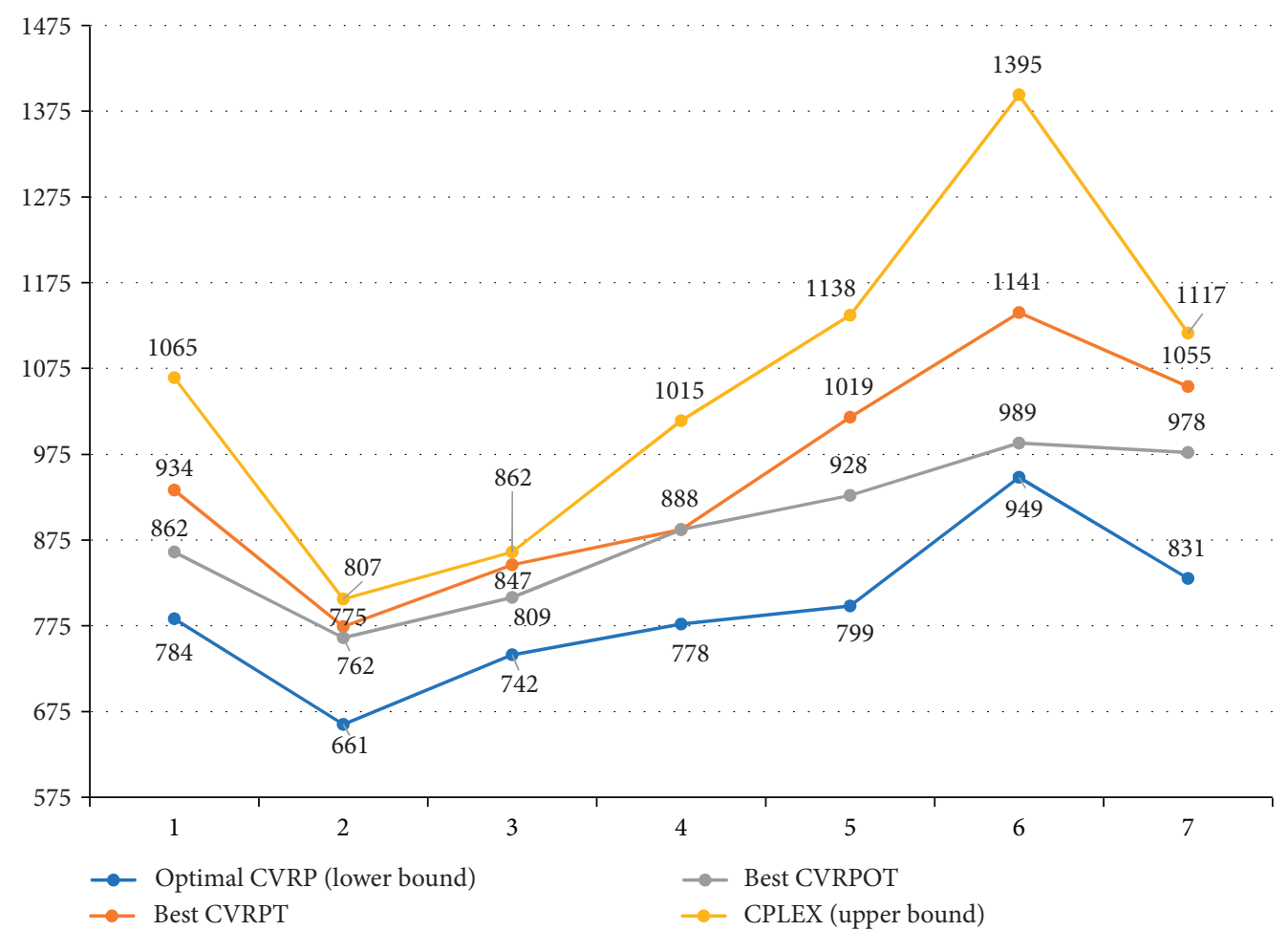

FIgURE 6: Result comparison of VRPT and VRPOT to the lower bound and upper bound.

TABle 6: Percentage of additional cost per unit of satisfied transshipment demand.

\begin{tabular}{lcc}
\hline Problem instance & \multicolumn{2}{c}{ \%Diff/TD } \\
& VRPT (\%) & VRPOT (\%) \\
\hline A n32 k5 & 3.19 & 3.32 \\
A n33 k5 & 2.87 & 3.06 \\
A n33 k6 & 2.36 & 1.81 \\
A n34 k5 & 2.02 & 2.02 \\
A n36 k5 & 3.93 & 2.69 \\
A n37 k5 & 3.63 & 2.77 \\
A n37 k6 & 2.89 & 1.05 \\
A n38 k5 & 3.73 & 2.29 \\
A n39 k5 & 1.72 & 1.51 \\
A n39 k6 & 3.37 & 2.53 \\
A n44 k6 & 2.82 & 2.55 \\
A n45 k6 & 3.52 & 2.73 \\
A n45 k7 & 2.02 & 2.31 \\
A n46 k7 & 2.74 & 1.81 \\
A n48 k7 & 3.89 & 2.22 \\
A n53 k7 & 3.53 & 2.94 \\
A n54 k7 & 2.17 & 2.07 \\
A n55 k9 & 1.73 \\
A n60 k9 & 2.35 & 2.08 \\
A n61 k9 & 2.68 & 1.92 \\
A n62 k8 & 2.11 & 2.17 \\
A n63 k9 & 2.95 & 1.90 \\
A n63 k10 & 1.64 & 2.57 \\
A n64 k9 & 2.54 & 1.59 \\
A n65 k9 & 1.90 \\
A n69 k9 & 3.19 & 1.90 \\
A n80 k10 & 2.64 & 1.60 \\
\hline
\end{tabular}

problem has to be solved six days per week in two sizes: 22store problem on Monday, Wednesday, and Friday and 15store problem on Tuesday, Thursday, and Saturday. These problem sizes can be effectively solved as a VRPOT by the proposed TA-NS heuristic with the forbidden list.

Expanding the problem to include all business units results in a large problem of 68 store locations. This problem should also be solved as CRPOT since it is more economical to satisfy only the transshipment demands that can be added to the regular demand without incurring much additional transportation distance (i.e., cost). The results from testing the TA-NS heuristic with forbidden list on the problem of this size in set A gives a solution with approximately $17 \%$ higher than the lower bound solution, while it can satisfy 9 out of 14 transshipment demands (64\%). From the small problem instance solution, for problem instances where three out of four transshipment demands $(75 \%$, which is close to $64 \%$ of the large problem) are satisfied (i.e., see rows 12-15 in Table 3), the percent off-optimal between the VRPOT and VRP (lower bound) is $13.95 \%$ and $14.14 \%$ for the CEN and OFF instances, respectively. Since for the problem of this size, (1) the TA-NS solution is approximately $17 \%$ higher than the lower bound and (2) the optimal solution of CVRPOT at a relatively close percent of transshipment demand satisfied is around 14\% higher than the lower bound, we can imply that the optimal solutions of VRPOT are closer to the TA-NS solution than the lower bound. Therefore, a practical implication can be made that the proposed heuristic for the large real problem is also effective. 


\section{Conclusions}

This paper presents the newly defined VRPT and its MILP model for small problem instances, as well as hybrid TA-NS heuristic for medium to large-scale problem instances. Small problem instances of 19 customer nodes are solved to optimality to assess the impact of satisfying the transshipment demands on the total distance. It was found that the optimal solutions of VRPT expectedly have longer distances than those of the VRP when transshipment demands are not satisfied. For VRPT, the more the transshipment demands are satisfied, the longer the additional distance they incur. There is clearly a trade-off between efficiency of delivery operation (traveling distance) and responsiveness (end customer service).

Further analysis of the optimal solutions of VRPT at various levels of transshipment demand reveals that some transshipment demands can be satisfied without incurring much additional distance, whereas some significantly increase the distance. This indicates that in practice, transshipment demands should be optional. As a result, the forbidden list of transshipment pairs is constructed, which can help to identify and eliminate the transshipment pairs that should not be in the same delivery routes. This is to prevent incurring too much additional distance from satisfying the transshipment demands.

The developed TA-NS algorithms, without and with implementing the forbidden list, are tested on small problem instances and medium to large problem instances of VRPT and VRPOT, respectively. Computational test results show that the TA-NS heuristic outperforms the CPLEX solver when the computation time for the CPLEX solver is limited to 3,600 seconds. The results demonstrate the effectiveness of the TA-NS heuristic and the forbidden list in identifying appropriate transshipment demands that should be satisfied. In practice, the depot has to set either the level of responsiveness (transshipment demand to satisfy) or efficiency (additional distance incurred) to obtain the desired result with respect to end customer service requirements and the resources required to satisfy the transshipment demands.

Future research directions are as follows: (1) extending the scope of the problem to include delivery time windows and (2) incorporating other real-world aspects to the problem, such as asymmetric distance between nodes or stochastic travel time depending on the traffic level during the time that vehicles are on the road.

\section{Data Availability}

The data used to support the findings of this study are available from the corresponding author upon request.

\section{Conflicts of Interest}

The authors declare that there are no conflicts of interest regarding the publication of this paper.

\section{References}

[1] M. Battarra, J.-F. Cordeau, and M. Iori, "Pickup-and-delivery problems for goods transportation," in Vehicle Routing: Problems, Methods, and Applications, P. Toth and D. Vigo, Eds., MOS-SIAM Series on Optimization The Society for Industrial and Applied Mathematics (SIAM) and the Mathematical Optimization Society (MOS), Philadelphia, PA, USA, 2nd edition, 2014.

[2] P. Toth and D. Vigo, Eds., MOS-SIAM Series on Optimization, The Society for Industrial and Applied Mathematics (SIAM) and the Mathematical Optimization Society (MOS), Philadelphia, PA, USA, 2014.

[3] C.-K. Ting, X.-L. Liao, Y.-H. Huang, and R.-T. Liaw, "Multivehicle selective pickup and delivery using metaheuristic algorithms," Information Sciences, vol. 406-407, pp. 146-169, 2017.

[4] D. Xu, K. Li, X. Zou, and L. Liu, "An unpaired pickup and delivery vehicle routing problem with multi-visit," Transportation Research Part E, vol. 103, pp. 218-247, 2017.

[5] A. S. Tasan and M. Gen, "A genetic algorithm based approach to vehicle routing problem with simultaneous pick-up and deliveries," Computers and Industrial Engineering, vol. 62, no. 3, pp. 755-761, 2012.

[6] F. P. Goksal, I. Karaoglan, and F. Altiparmak, "A hybrid discrete particle swarm optimization for vehicle routing problem with simultaneous pickup and delivery," Computers and Industrial Engineering, vol. 65, no. 1, pp. 39-53, 2013.

[7] M. Avci and S. Topaloglu, "An adaptive local search algorithm for vehicle routing problem with simultaneous and mixed pickups and deliveries," Computers and Industrial Engineering, vol. 83, pp. 15-29, 2015.

[8] I. Kucukoglu and N. Ozturk, "An advanced hybrid metaheuristic algorithm for the vehicle routing problem with backhauls and time windows," Computers and Industrial Engineering, vol. 86, pp. 60-68, 2015.

[9] M. Avci and S. Topaloglu, "A hybrid metaheuristic algorithm for heterogeneous vehicle routing problem with simultaneous pickup and delivery," Expert Systems with Applications, vol. 53, pp. 160-171, 2016.

[10] N. Wassan, N. Wassan, G. Nagy, and S. Salhi, "The multiple trip vehicle routing problem with backhauls: formulation and a two-level variable neighbourhood search," Computers and Operations Research, vol. 78, pp. 454-467, 2017.

[11] S. Reil, A. Bortfeldt, and L. Mönch, "Heuristics for vehicle routing problems with backhauls, time windows, and 3D loading constraints," European Journal of Operational Research, vol. 266, no. 3, pp. 877-894, 2018.

[12] N. A. Wassan, G. Nagy, and S. Ahmadi, "A heuristic method for the vehicle routing problem with mixed deliveries and pickups," Journal of Scheduling, vol. 11, no. 2, pp. 149-161, 2008.

[13] G. Gong, Q. Deng, X. Gong, L. Zhang, H. Wang, and H. Xie, "A bee evolution algorithm for multiobjective vehicle routing problem with simultaneous pickup and delivery," Mathematical Problems in Engineering, vol. 2571380, 2018.

[14] I. Gribkovskaia, G. Laporte, and A. Shyshou, "The single vehicle routing problem with deliveries and selective pickups," Computers and Operations Research, vol. 35, no. 9, pp. 2908-2924, 2008.

[15] O. Polat, "A parallel variable neighborhood search for the vehicle routing problem with divisible deliveries and 
pickups," Computers and Operations Research, vol. 85, pp. 71-86, 2017.

[16] C. Archetti and M. G. Speranza, "Vehicle routing problems with split deliveries," International Transactions in Operational Research, vol. 19, no. 1-2, pp. 3-22, 2012.

[17] N. A. Wassan and G. Nagy, "Vehicle routing problem with deliveries and pickups: modelling issues and meta-heuristics solution approaches," International Journal of Transportation, vol. 2, no. 1, pp. 95-110, 2014.

[18] Y. Qu and J. F. Bard, "A GRASP with adaptive large neighborhood search for pickup and delivery problems with transshipment," Computers and Operations Research, vol. 39, no. 10, pp. 2439-2456, 2012.

[19] S. N. Parragh and V. Schmid, "Hybrid column generation and large neighborhood search for the dial-a-ride problem," Computers and Operations Research, vol. 40, no. 1, pp. 490497, 2013.

[20] D. Kirchler and R. Wolfler Calvo, "A granular tabu search algorithm for the dial-a-ride problem," Transportation Research Part B: Methodological, vol. 56, pp. 120-135, 2013.

[21] R. Masson, F. Lehuede, and O. Peton, "The dial-a-ride problem with transfers," Computers \& Operations Research, vol. 41, pp. 12-23, 2014.

[22] A. Rais, F. Alvelos, and M. S. Carvalho, "New mixed integerprogramming model for the pickup-and-delivery problem with transshipment," European Journal of Operational Research, vol. 235, no. 3, pp. 530-539, 2014.

[23] S. Madankumar and C. Rajendran, "Mathematical models for green vehicle routing problems with pickup and delivery: a case of semiconductor supply chain," Computers and Operations Research, vol. 89, pp. 183-192, 2018.

[24] M. Soysal, M. Cimen, and E. Demir, "On the mathematical modeling of green one-to-one pickup and delivery problem with road segmentation," Journal of Cleaner Production, vol. 174, pp. 1664-1678, 2018.

[25] C. Mues and S. Pickl, "Transshipment and time windows in vehicle routing," in Proceedings of the 8th International Symposium on Parallel Architectures, Algorithms and Networks (ISPAN'05), Las Vegas, NV, USA, December 2005.

[26] F.-M. Yang and H.-J. Xiao, "Models and algorithms for vehicles routing problems with transshipment centers," Systems Engineering-Theory and Practice, vol. 27, no. 3, pp. 28-35, 2007.

[27] L. C. Coelho, J.-F. Cordeau, and G. Laporte, "The inventoryrouting problem with transshipment," Computers and Operations Research, vol. 39, no. 11, pp. 2537-2548, 2012.

[28] A. Azadeh, S. Elahi, M. H. Farahani, and B. Nasirian, "A genetic algorithm-Taguchi based approach to inventory routing problem of a single perishable product with transshipment," Computers and Industrial Engineering, vol. 104, pp. 124-133, 2017.

[29] M. Drexl, "Applications of the vehicle routing problem with trailers and transshipments," European Journal of Operational Research, vol. 227, no. 2, pp. 275-283, 2013.

[30] M. Drexl, "Branch-and-cut algorithms for the vehicle routing problem with trailers and transshipments," Networks, vol. 63, no. 1, pp. 119-133, 2014.

[31] H. Ahmad, P. Hamzah, Z. A. M. M. Yasin, and S. S. R. Shariff, "Location routing inventory problem with transshipment (LRIP-T)," in Proceedings of the 2014 International Conference on Industrial Engineering and Operations Management, Bali, Indonesia, January 2014.
[32] Y. Wang, X. Ma, Z. Li et al., "Profit distribution in collaborative multiple centers vehicle routing problem," Journal of Cleaner Production, vol. 144, pp. 203-219, 2017.

[33] H. Fan, J. Wu, X. Li, and X. Jiang, "Presenting a multi-start hybrid heuristic for solving the problem of two-echelon location-routing problem with simultaneous pickup and delivery (2E-LRPSPD)," Journal of Advanced Transportation, vol. 2020, Article ID 9743841, 23 pages, 2020.

[34] Y. Wang, S. Peng, X. Zhou, M. Mahmoudi, and L. Zhen, "Green logistics location-routing problem with eco-packages," Transportation Research Part E: Logistics and Transportation Review, vol. 143, Article ID 102118, 2020.

[35] Y. Wang, Y. Yuan, X. Guan et al., "Collaborative two-echelon multicenter vehicle routing optimization based on statespace-time network representation," Journal of Cleaner Production, vol. 258, Article ID 120590, 2020.

[36] M. Gansterer, R. F. Hartl, and P. E. H. Salzmann, "Exact solutions for the collaborative pickup and delivery problem," Central European Journal of Operational Research, vol. 26, no. 2, pp. 357-371, 2018.

[37] G. Laporte, "What you should know about the vehicle routing problem," Naval Research Logistics, vol. 54, no. 8, pp. 811-819, 2007.

[38] B. E. Gillett and L. R. Miller, "A heuristic algorithm for the vehicle-dispatch problem," Operations Research, vol. 22, no. 2, pp. 340-349, 1974.

[39] G. Clarke and J. W. Wright, "Scheduling of vehicles from a central depot to a number of delivery points," Operations Research, vol. 12, no. 4, pp. 568-581, 1964.

[40] P. Augerat, J. M. Belenguer, E. Benavent, A. Corberán, and G. Rinaldi, "Computational results with a branch and cut code for the capacitated vehicle routing problem," Tech. Rep. 1 RR949-M, ARTEMIS- IMAG, Grenoble France, 1995. 